\title{
Mixed Adenoneuroendocrine Carcinoma of the Perianal Region
}

\author{
Jelena Berendika ${ }^{1}$, Gordan Nikic ${ }^{1}$, Dejan -Dokanović ${ }^{1}$, Milka Vještica ${ }^{1}$, Sanja Savić ${ }^{1}$, Saša \\ Jungić $^{1}$, Zdenka Gojković ${ }^{\text {, Božana Babić }}{ }^{\text {, and Biljana Tubic }}{ }^{2}$ \\ ${ }^{1} \mathrm{UKC} \mathrm{RS}$ \\ ${ }^{2}$ University of Banja Luka
}

March 9, 2021

\begin{abstract}
Introduction: Mixed adenoneuroendocrine carcinoma (MANEC) is a tumor of the gastrointestinal tract that contains both exocrine and endocrine components, with each component representing a minimum of $30 \%$ of the total tumor mass.
\end{abstract}

\section{Introduction}

MANEC is a neoplasm characterized by significant histological heterogeneity and is characterized by the simultaneous presence of both adenocarcinomatous and neuroendocrine differentiation; their definition includes each component found in at least $30 \%$ of the tumor. (1) They most commonly arise in the colon, appendix, rectum or stomach, however, a limited number of MANECs have been reported to originate in the gallbladder. (2) Due to the low frequency of this histotype, only a small number of cases have been described, and there is no established therapeutic strategy. Here we present a case of the patient with MANEC of anal region.

\section{Case Illustration}

The 75-year-old female presented with nodul in the perianal region that has been present for several months. DX was atheroma and excision of the tumor was performed. Pathology report confirmed MIXED ADENONEUROENDOCRINE CARCINOMA-MANEC. Additional immunohistochemistry analysis was performed on the examined sample: Synaptophysin-positive, CK 20-positive, CK AE1 / AE3-positive, CK7negative, CA 125-negative. (Figure 1 )

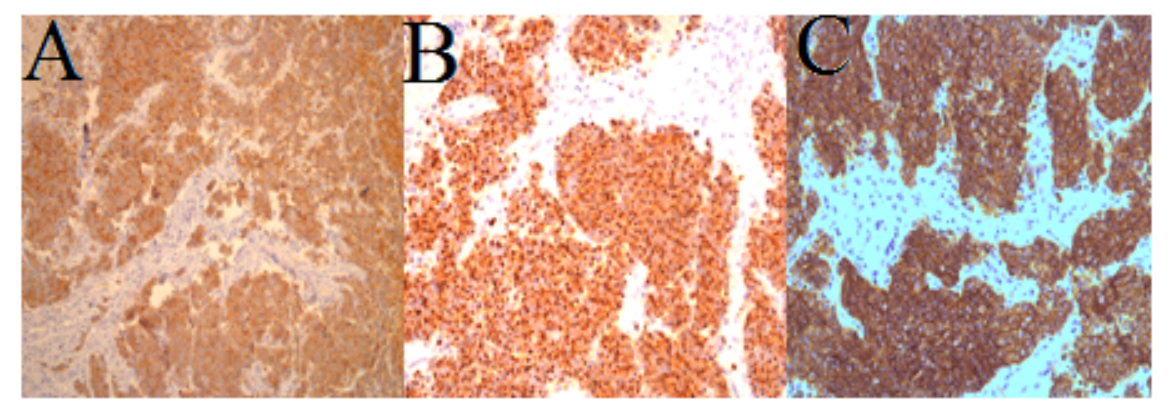

Firugre 1 : Magnification of 40x, staining with : Synaptophysin-positive (A), CK 20-positive (B), CK AE1 / AE3-positive (C) 
After initial excision patient was not reffered to an oncologist. After two months she developed symptoms of an infection and pain in the anal region and was admitted to a hospital. She was operated on and extirpation of the reamining tumor was performed. Pathohistological examination of sample was described as: ADENOCARCINOMA (GRADUS III). Immunohistochemistry: CK PAN-positive, CK 20-positive, S100-negative, VIMENTIN-negative, LCA-negative, CK7-negative, CK 5/6-negative.

She was refferd to the oncologist, two months after the initial diagnosis. At first appointment her ECOG PS was 0 . An ultrasound examination (US) of the abdomen, small pelvis and inguinal regions was performed and it confirmed metastatic disease ; left iliac lymph node $4.7 \mathrm{~cm}$ in size and a conglomerate of pathologicaly altered lymph nodes in the ingunal region on the left $10 \times 8 \mathrm{~cm}$ in size. A biopsy was performed and the pathological report showed that the sample morphologically and immunohistochemically corresponds to the previously diagnosed tumor: MIXED ADENONEUROENDOCRINE CARCINOMAMANEC, Synaptophysin-positive, Chromogranin A-positive, CD56-positive, CK 20-positive, NSE-positive, CK7-negative, Ki67-about $90 \%$ of tumor cells show nuclear positivity. Patient was presented to the MDT and the desicion was made to start with PE regimen in the first line of treatment. After two cycles, there was an evident clinical progression and she started to have difficulty walking Lab reports showed elevated levels of CYFRA 21-1 $5.3 \mathrm{ng} / \mathrm{ml}$, LDH $444 \mathrm{U} / \mathrm{L}$ and NSE 140,2 ng / ml. Progression-free survival (PFS) for the first-line of chemotherapy was 1 month. In the second line patient was treated with paclitaxel and ifosfamide (PI) combination chemotherapy.After three cycles of therapy initial responce was stable disease . Patient progressed after six cycles CT showed enlargement of the mass in the left ingunal region, now 10x9x6.5 cm in size, mass in the pelvis now $10 \mathrm{~cm}$ in size (Figure 2), onset of peritoneal carcinomatosis and cutaneous and subcutaneous metastatic deposits. She had worsening of her previous condition, limited movement in her left leg and developed DVT . She started LMWH for treatment of DVT. Her ECOG PS was 3.PFS for second-line of therapy was 4 months.

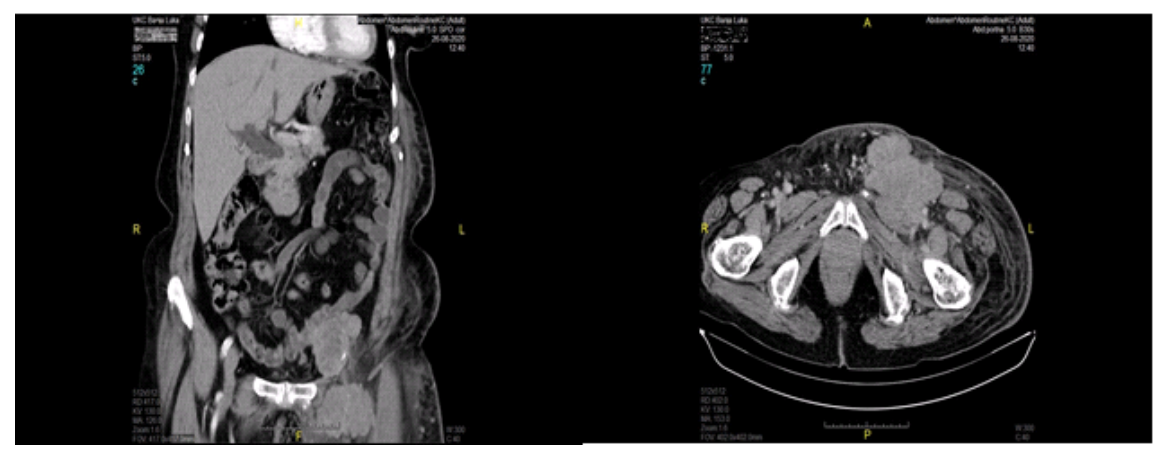

Figure 2 : Computed tomography showed enlarged conglomerates lgl inguinal left 


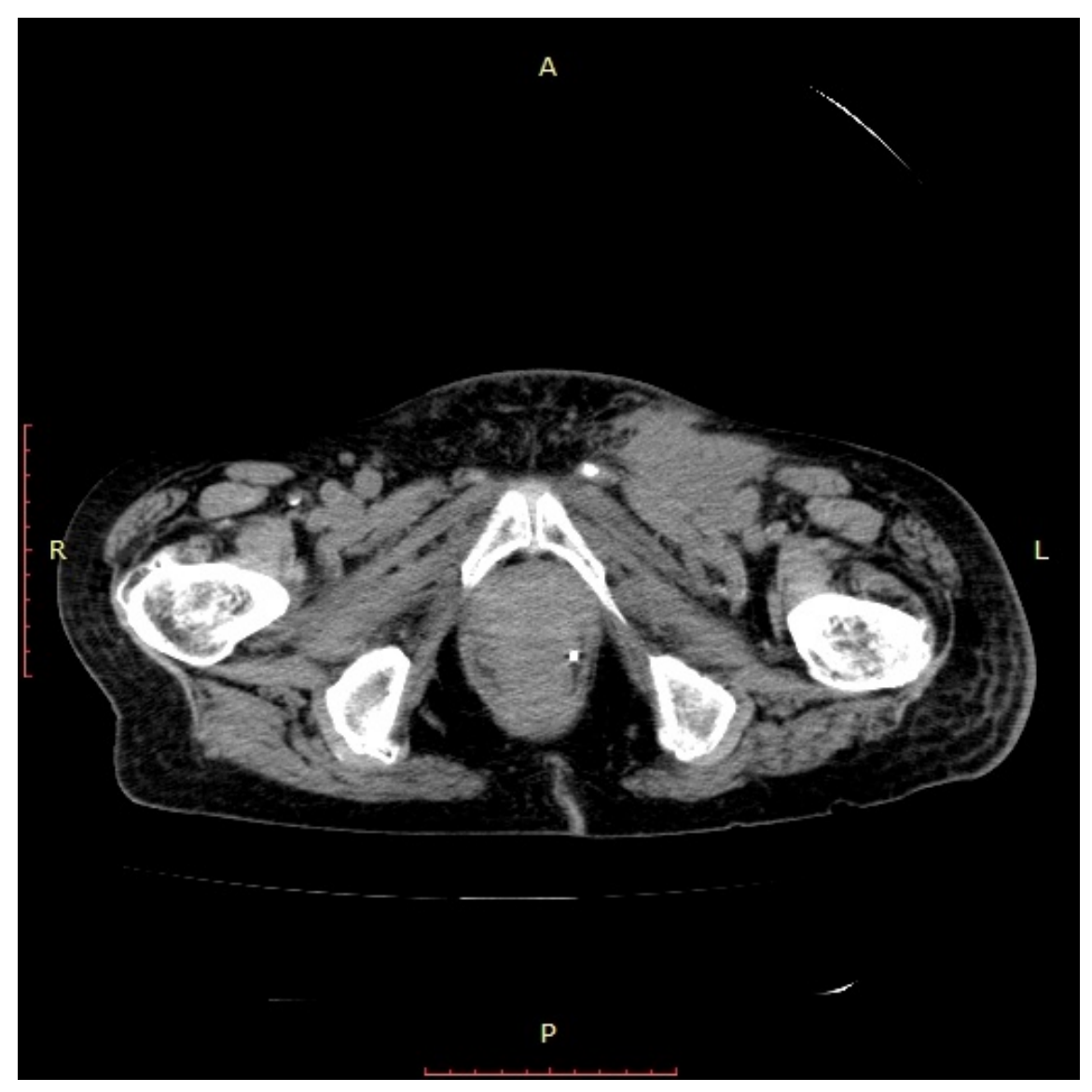

Patient was presented to the MDT again and started third line chemotherapy with FOLFIRI3 regimen plus Sandostatin LAR After two cycles NSE levels dropped to $16.7 \mathrm{ng} / \mathrm{ml}$ and LDH levels were nomal. After six cycles of FOLFIRI3 regimen with Sandostatin LAR applied every other cycle, CT showed tumor regression, with mass in the left inguninum now $7 \times 5 \mathrm{~cm}$ in size (Figure 3 ) and no other enlarged lymph nodes . NSE was $5.8 \mathrm{ng} / \mathrm{ml}$. Swelling in her leg went down and her condition improved. ECOG PS is 1 . She is still in treatment .

Figure 3 : Computed tomography showed regression of lgl conglomerate inguinal left

\section{Discussion}

Our patient developed cancer at the age of 75 . The primary treatment option is surgery and in the advanced stage of the disease, due to the small number of cases, there are no recommendations for systemic therapy . Examples of chemotherapy based on platinum preparations (3), fluorouracils (4) and taxanes are given in the literature. In our case, the patient had a positive response only to the third line of chemotherapy, FOLFIRI3 regimen plus Sandostatin LAR, which emphasizes the importance of early evaluation of the clinical response and changes in chemotherapy at the first progression of the disease. No data on the radiosensitivity of this tumor were found in the reviewed literature, so we did not decide to use radiotherapy in the treatment of our patient.

\section{Conclusion}

MANEC is very rare, aggressive, rapidly spreading disease, usually with poor prognosis. (5). Although a rare cancer, MANEC presents a significant therapeutic challenge because there are no clear guidelines based on randomized trials. In our case, the patient responded well to the third line of chemotherapy, FOLFIRI3 
regimen plus Sandostatin LAR . It is of great importance the early recognition of tumor rarities such as MANEC, the multidisciplinary approach from the beginning of treatment as well as the need to form a single reference center for rare tumors.

\section{References}

1. Paspala A, Machairas N, Prodromidou A, Spartalis E, Ioannidis A, Kostakis ID, Papaconstantinou D, Nikiteas N. Management of MANEC of the colon and rectum: A comprehensive review of the literature. Mol Clin Oncol. 2018 Aug;9(2):219-222. doi: 10.3892/mco.2018.1649. Epub 2018 Jun 11. PMID: 30101026; PMCID: PMC6083408.

2. Machairas N, Paspala A, Frountzas M, Tsilimigras DI, Moris D, Ntomi V, Tsapralis D, Schizas D. Mixed Adenoneuroendocrine Carcinoma (MANEC) of the Gallbladder: A Systematic Review of Outcomes Following Surgical Management. In Vivo. 2019 Nov-Dec;33(6):1721-1726. doi: 10.21873/invivo.11662. PMID: 31662496; PMCID: PMC6899129.

3. Moyón Constante MA, Moyón Constante FX, Tufiño JF, Cárdenas Patiño A, Molina GA, Gutierrez BM. Gastric mixed adenoneuroendocrine carcinoma case report. SAGE Open Med Case Rep. 2019 Feb 8;7:2050313X19828918. doi: 10.1177/2050313X19828918. PMID: 30800309; PMCID: PMC6378457.

4. Cherbanyk F, Gassend JL, Dimitrief M, Andrejevic-Blant S, Martinet O, Pezzetta E. A Rare Type of Colorectal Cancer: Mixed Adeno-Neuroendocrine Carcinoma (MANEC). Chirurgia (Bucur). 2017 Mar-Apr;112(2):152-156. doi: 10.21614/chirurgia.112.2.152. PMID: 28463674

5. Dulskas A, Pilvelis A. Oncologic outcome of mixed adenoneuroendocrine carcinoma (MANEC): A single center case series. Eur J Surg Oncol. 2020 Jan;46(1):105-107. doi: 10.1016/j.ejso.2019.08.002. Epub 2019 Aug 2. PMID: 31444026. 